\title{
A CHARACTERISATION OF $C^{*}$-ALGEBRAS
}

\author{
by M. A. HENNINGS
}

(Received 24th March 1986, revised 10th June 1986)

\section{Introduction}

It is of some interest to the theory of locally convex *-algebras to know under what conditions such an algebra $A$ is a pre- $C^{*}$-algebra (the topology of $A$ can be described by a submultiplicative norm such that $\left\|x^{*} x\right\|=\|x\|^{2}, \forall x \in A$ ). W.e recall that a locally convex *-algebra is a complex *-algebra $A$ with the structure of a Hausdorff locally convex topological vector space such that the multiplication is separately continuous, and the involution is continuous.

Allan [3] has studied the problem for normed algebras, showing that a unital Banach *-algebra is a $C^{*}$-algebra if and only if $A$ is symmetric and the set $\mathscr{B}$ of all absolutely convex hermitian idempotent closed bounded subsets of $A$ has a maximal element. Recalling that an element $x$ of a locally convex algebra $A$ is bounded if there exists $\lambda>0$ such that the set $\left\{(\lambda x)^{n}: n \in \mathbb{N}\right\}$ is bounded, we find the following simple generalisation of Allan's result:

Proposition 0. A unital locally convex *-algebra $A$ is a $C^{*}$-algebra if and only if:

(a) $A$ is sequentially complete;

(b) $A$ is barreled;

(c) every element of $A$ is bounded;

(d) $A$ is symmetric;

(e) the family $\mathscr{B}$ of absolutely convex hermitian idempotent closed bounded subsets of $A$ has a maximal element $B_{0}$.

Proof. For any $x=x^{*}$ in $A$, we can find $\lambda>0$ such that the closed absolutely convex hull of the set $\left\{(\lambda x)^{n}: n \in \mathbb{N}\right\}$ is contained in $B_{0}$. Thus $B_{0}$ is absorbing, hence a barrel. Thus $B_{0}$ is a bounded neighbourhood of 0 , so the Minkowski functional $\|\cdot\|$ of $B_{0}$ is a norm on $A$ defining the original topology, and $(A,\|\cdot\|)$ is a Banach *-algebra satisfying the conditions of Theorem 2 of Allan [3].

Another interesting characterisation of $C^{*}$-algebras is to be found in the Vidav-Palmer Theorem (Bonsall and Duncan [4]), which states that a unital Banach algebra can be given an involution which turns it into a $C^{*}$-algebra if and only if $A=H(A)+i H(A)$, 
where

$$
H(A)=\left\{h \in A: f(h) \in \mathbb{R} \forall f \in A^{\prime} \text { s.t. } f(e)=\|f\|=1\right\}
$$

Unlike that of Allan, this approach does not lend itself so well to generalisation to the non-normed case. However Wood [12] has proved an analogue of the Vidav-Palmer Theorem, which uses the same principle of numerical range to characterise a particular class of locally convex *-algebras (which contains all $C^{*}$-algebras), the so-called complete semi-GB*-algebras with hypocontinuous involution. $A$ semi- $G B^{*}$-algebra with continuous involution is a $G B^{*}$-algebra in the sense of Dixon [6]. Thus the VidavPalmer Theorem can be generalised to characterise $G B^{*}$-algebras, if not $C^{*}$-algebras.

In this paper we shall find a new characterisation of pre-C*-algebras in terms of properties of the positive elements $A^{+}$of the locally convex *-algebra $A$ and the continuous positive linear functionals $P(A)$ on $A . A^{+}$will be defined to be the closed algebraic cone, as used by Alcantara and Dubin [1] and other quantum field theorists; we shall not need to use the spectral theory of Allan [2]. The main property that we shall use (in the unital case) is an order-boundedness property concerning the absorption of positive elements by the order interval $[0, e]$. We shall prove that a unital locally convex *-algebra with identity $e$ is a $C^{*}$-algebra if and only if:

(a) $A$ is sequentially complete;

(b) $A$ is barreled;

(c) $A^{+}$is a normal cone for $A$;

(d) for $x \in A$ there exists $\lambda>0$ such that $e-\lambda x^{*} x \in A^{+}$,

and a similar characterisation for non-unital algebras will also be found.

Thanks go to Dr. D. A. Dubin for some interesting ideas, and for advice with the terminology and notation.

\section{Introduction of notation}

Let $(A, t)$ be a locally convex *-algebra. A natural candidate for the cone of positive elements of $A$ is given by $A^{+}$- the closed algebraic cone generated by the elements $\left\{x^{*} x: x \in A\right\}$. It is clear that $A^{+} \subseteq A_{h}$, the set of hermitian elements of $A$, and that $A_{h}$ is a real Hausdorff locally convex space with the induced topology.

If $A^{\prime}$ is the topological dual of $(A, t)$, we define the positive functionals $P(A)$ to be

$$
P(A)=\left\{f \in A^{\prime}: f\left(x^{*} x\right) \geqq 0 \forall x \in A\right\} .
$$

Applying the Cauchy-Schwarz inequality and the Hahn-Banach Theorem, we see that:

Lemma 1.

(a) $f\left(x^{*} y\right)=\overline{f\left(y^{*} x\right)}$ $f \in P(A), \quad x, y \in A$

(b) $\left|f\left(x^{*} y\right)\right|^{2} \leqq f\left(x^{*} x\right) f\left(y^{*} y\right) \quad f \in P(A), \quad x, y \in A$;

(c) if $x \in A_{h}$, then $x \in A^{+}$if and only if $f(x) \geqq 0$ for all $f \in P(A)$. 
Corollary 2. $P(A)=\{0\}$ if and only if $A^{+}=A_{h}$.

If $A$ is unital with identity $e$, then $A^{+}$is generating, every element of $P(A)$ is hermitian, and

$$
|f(x)|^{2} \leqq f(e) f\left(x^{*} x\right) \quad f \in P(A), \quad x \in A,
$$

so that $P(A)=\{0\}$ if and only if $-e \in A^{+}$(cf. Ky Fan [8, Theorem 1]). In this paper we shall at the least assume that $P(A)$ separates points of $A$. This is equivalent to saying that $P(A)$ spans a dense linear subspace of the weak dual $A_{\sigma}^{\prime}$ of $A$, or that $A^{+}$is a proper cone in $A$.

Lemma 3. If $A^{+}$is a proper cone in $A$, then $x \in A_{h}$ if and only if $f(x) \in \mathbb{R}$ for all $f \in P(A)$. Also $x^{*} x=0$ implies $x=0$.

It will also be necessary sometimes to assume that $P(A)$ is generating. When $(A, t)$ is barreled, this is equivalent to saying that $A^{+}$is a normal cone in $A$ (Schaefer [11, V. 3.4]).

\section{Infrabarreled spaces}

As in the proof of Proposition 0, we shall need to include a property akin to barreledness to characterise pre- $C^{*}$-algebras. However, although every Fréchet space (and hence every $C^{*}$-algebra) is barreled (Schaefer [11, II.7.1]), not every metrisable locally convex space is. For example (Schaefer [11, p. 70, Ex. 14]), if we let $X$ denote the subspace of $C[0,1]$ consisting of functions $f$ which vanish on a neighbourhood (depending on $f$ ) of 0 , then $X$ with the uniform norm is a pre- $C^{*}$-algebra which is not barreled. Thus, when characterising pre- $C^{*}$-algebras, barreledness is too strong a property.

Let $X$ be a locally convex space. A barrel in $X$ is called bound-absorbing if it absorbs every bounded subset of $X$, and we recall that $X$ is called infrabarreled if every boundabsorbing barrel is a neighbourhood of 0 . Every bornological space (and hence every pre- $C^{*}$-algebra) is infrabarreled. The relationship between bounded sets and null sequences found in 1.5 .3 of Schaefer [11] enables us to simplify the criterion for infrabarreledness to a form which we shall find more useful. We shall say that a barrel is null-absorbing if it absorbs every null sequence.

Lemma 4. A barrel is null-absorbing if and only if it is bound-absorbing.

Proof. If $U$ is a barrel in $X$ which is not bound-absorbing, let $B$ be a bounded set not absorbed by $U$. Thus we can find a sequence $\left(x_{n}\right)$ in $B$ such that $x_{n} \notin n^{2} U$ for all $n \in \mathbb{N}$. Thus $\left(n^{-1} x_{n}\right)$ is a null sequence. If $n^{-1} x_{n} \in \lambda U$ for all $n \in \mathbb{N}$ and some $\lambda>0$, it would follow that $x_{n} \in n^{2} U$ for all $n \geqq \lambda$. This contradiction implies that $\left(n^{-1} x_{n}\right)$ is not absorbed by $U$, and so $U$ is not null-absorbing. The converse follows since every null sequence is bounded.

Corollary 5. A locally convex space $X$ is infrabarreled if and only if every nullabsorbing barrel is a neighbourhood of 0 . 


\section{Properties of pre- $C^{*}$-algebras}

In this section we shall list some properties which are common to all pre- $C^{*}$-algebras. In the next section we shall prove that these properties in fact characterise pre- $C^{*}$. algebras.

Let $A$ be a pre- $C^{*}$-algebra, and let $B$ be its $C^{*}$-algebra completion. If we define the positive elements $B^{+}$and the positive linear functionals $P(B)$ of $B$ as above, then $P(B)$ is generating (Sakai [10, Proposition 1.17.1]), so that $B^{+}$is a normal cone in $B$, and:

\section{Lemma 6.}

(a) $A^{+}=B^{+} \cap A$;

(b) if $F \in P(B)$, then $\left.F\right|_{A} \in P(A)$;

(c) if $f \in P(A)$, we can find a unique element $F \in P(B)$ which extends $f$.

Corollary 7. $P(A)$ is generating.

Finally, if $A$ is unital with identity $e$, we obtain the following result:

Proposition 8. If $\left(x_{n}\right)$ is a null sequence in $A$, we can find $\lambda>0$ such that $e-\lambda x_{n}^{*} x_{n} \in A^{+}$ for all $n \in \mathbb{N}$.

Proof. We can find $K>0$ such that $\left\|x_{n}^{*} x_{n}\right\|=\left\|x_{n}\right\|^{2} \leqq K$ for all $n$, and hence $e-K^{-1} x_{n}^{*} x_{n}$ belongs to $B^{+} \cap A=A^{+}$for all $n$.

Thus, if $A$ is a unital pre- $C^{*}$-algebra, then $A$ is a unital locally convex *-algebra such that:

(A) $\boldsymbol{A}$ is infrabarreled;

(B) $P(A)$ is generating;

(C) for any null sequence $\left(x_{n}\right)$ in $A$, we can find $\lambda>0$ such that $e-\lambda x_{n}^{*} x_{n} \in A^{+}$for all $n \in \mathbb{N}$.

\section{A characterisation of pre- $C^{*}$-algebras}

We shall now show that the properties $(\mathrm{A}),(\mathrm{B}),(\mathrm{C})$ characterise the pre- $C^{*}$-algebras over all unital locally convex *-algebras. Initially, however, we begin by weakening property (B). Let us therefore assume that $(A, t)$ is a unital locally convex *-algebra which satisfies properties (A), (C) and

(B') $A^{+}$is a proper cone in $A$.

We need to find a bounded neighbourhood of 0 in $A$ whose Minkowski functional defines a $C^{*}$-algebra norm on $A$. To this end we define the set

$$
V=\left\{x \in A: e-x^{*} x \in A^{+}\right\}
$$

Theorem 9. $V$ is an idempotent barrel in $A$. 
Proof. For any $x \in A$, considering the null sequence $\left(x_{n}\right)$ defined by $x_{n}=n^{-1} x$ shows us that $V$ is absorbing. An elementary application of the Cauchy-Schwarz inequality (Lassner [9]) and Lemma 1 shows that

$$
V=\bigcap_{f \in P(A)} \bigcap_{y \in A}\left\{x \in A:\left|f\left(y^{*} x\right)\right|^{2} \leqq f\left(y^{*} y\right) f(e)\right\},
$$

and hence $V$ is absolutely convex and closed. Thus $V$ is a barrel.

For any $y \in A$ and $f \in P(A)$ we can define $f_{y} \in P(A)$ by $f_{y}(x)=f\left(y^{*} x y\right) \quad(x \in A)$. If $x, y \in V$, then for any $f \in P(A)$ we have $f\left((x y)^{*}(x y)\right)=f_{y}\left(x^{*} x\right) \leqq f_{y}(e)=f\left(y^{*} y\right) \leqq f(e)$, so that $e-(x y)^{*}(x y) \in A^{+}$, and hence $x y \in V$. Thus $V$ is idempotent.

Corollary 10. $V$ is a neighbourhood of 0 in $A$, and so the Minkowski functional $\|x\|=$ $\inf \{\lambda>0: x \in \lambda V\}(x \in A)$ of $V$ is a continuous submultiplicative seminorm on $A$.

Proof. Condition (C) states precisely that $V$ is null-absorbing.

Proposition 11. If $x \in A$ and $\lambda \geqq 0$, then $f\left(x^{*} x\right) \leqq \lambda^{2} f(e)$ for all $f \in P(A)$ if and only if $\lambda \geqq\|x\|$. Thus

$$
|f(x)| \leqq f(e)\|x\| \quad f \in P(A), \quad x \in A,
$$

and so $\|\cdot\|$ is a norm on $A$, and every element of $P(A)$ is a continuous linear functional on the normed space $(A,\|\cdot\|)$.

Proof. $f\left(x^{*} x\right) \leqq \lambda^{2} f(e) \forall f \in P(A) \Leftrightarrow f\left(x^{*} x\right) \leqq(\lambda+\varepsilon)^{2} f(e) \forall f \in P(A), \forall \varepsilon>0$

$$
\begin{aligned}
& \Leftrightarrow e-\left[(\lambda+\varepsilon)^{-1} x\right]^{*}\left[(\lambda+\varepsilon)^{-1} x\right] \in A^{+} \forall \varepsilon>0 \\
& \Leftrightarrow x \in(\lambda+\varepsilon) V \forall \varepsilon>0 \\
& \Leftrightarrow\|x\| \leqq \lambda+\varepsilon \Leftrightarrow\|x\| \leqq \lambda
\end{aligned}
$$

(using Lemma 1). Thus $f\left(x^{*} x\right) \leqq\|x\|^{2} f(e)$ for all $f \in P(A)$ and $x \in A$, and so (3) yields (5). Since $A^{+}$is a proper cone, (5) implies that $\|\cdot\|$ is a norm.

Proposition 12. $\left\|x^{*} x\right\|=\|x\|^{2}$ for all $x \in A$, so that $(A,\|\cdot\|)$ is a pre-C*-algebra.

Proof. For any $f \in P(A)$ we see that

$$
\begin{aligned}
f\left(\left(x^{*} x\right)^{*}\left(x^{*} x\right)\right)^{2} & =f\left(x^{*} x x^{*} x\right)^{2} \leqq f\left(x^{*} x\right) f\left(x^{*} x x^{*} x x^{*} x\right) \leqq\|x\|^{2} f(e) f_{x^{*} x}\left(x^{*} x\right) \\
& \leqq\|x\|^{4} f(e) f_{x^{*} x}(e)=\|x\|^{4} f(e) f\left(x^{*} x x^{*} x\right) .
\end{aligned}
$$

Thus $f\left(\left(x^{*} x\right)^{*}\left(x^{*} x\right)\right) \leqq\|x\|^{4} f(e)$ for all $f \in P(A)$, and so $\left\|x^{*} x\right\| \leqq\|x\|^{2}$. But (5) implies that $f\left(x^{*} x\right) \leqq\left\|x^{*} x\right\| f(e)$ for all $f \in P(A)$, so that $\|x\|^{2} \leqq\left\|x^{*} x\right\|$. 
Thus, if $(A, t)$ is a unital locally convex *-algebra satisfying (A), ( $\left.\mathrm{B}^{\prime}\right),(\mathrm{C})$, then we can define a pre- $C^{*}$-algebra topology $T$ on $A$ which is coarser than $t$. Replacing $\left(\mathrm{B}^{\prime}\right)$ by (B) enables us to sharpen the result.

Proposition 13. If $(A, t)$ satisfies properties (A),(B),(C), then $T=t$, so that $(A, t)$ is a pre-C*algebra.

Proof. Since $t \geqq T$, every element of the norm dual $A^{\sim}$ of $(A, T)$ belongs to $A^{\prime}$. But $P(A)$ is generating, and every element of $P(A)$ belongs to $A^{\sim}$. Thus $A^{-}=A^{\prime}$, and so $\tau\left(A, A^{\prime}\right) \geqq t \geqq T \geqq \sigma\left(A, A^{\prime}\right)$. Since $V$ is the closed unit ball of $(A, T)$, it is $T$-bounded, and hence $t$-bounded (Schaefer [11, IV.3.3]). Thus $T \geqq t$, and so $T=t$.

Hence, summarising the results of the last two sections, we see that:

Theorem 14. If $A$ is a unital locally convex *-algebra, then $A$ is a pre-C*-algebra if and only if it satisfies properties (A),(B),(C).

The order-boundedness property $(C)$ is fairly complicated. We might like to simplify it by replacing $(C)$ by the property

(C') for any $x \in A$, we can find $\lambda>0$ such that $e-\lambda x^{*} x \in A^{+}$.

Examination of the proof of Theorem 9 shows that $V$ is still an idempotent barrel, but is no longer necessarily null-absorbing. Thus, if we wish to replace property $(C)$ by property $\left(C^{\prime}\right)$, we need to strengthen property $(A)$.

Theorem 15. If $(A, t)$ is a unital locally convex *-algebra such that:

(A') $A$ is barreled;

(B') $A^{+}$is a proper cone in $A$;

(C') for any $x \in A$ we can find $\lambda>0$ such that $e-\lambda x^{*} x \in A^{+}$,

then we can find a pre-C*-algebra topology $T$ on $A$ which is coarser than $t$.

Corollary 16. A unital locally convex *algebra $(A, t)$ is a barreled pre-C*-algebra if and only if it satisfies properties $\left(\mathrm{A}^{\prime}\right),(\mathrm{B}),\left(\mathrm{C}^{\prime}\right)$.

\section{Algebras without identity}

It would be useful to generalise the results of Section 5 to cover the case of algebras without identity. Evidently, property (C) would have to be changed, as it is explicitly dependent on an identity element $e$.

If $A$ is a locally convex *-algebra without identity, we can form the unital algebra $A_{e}=A \oplus \mathbb{C} e$ in the usual way (Allan [2]), giving it the product topology. Our first important observation is that the property of infrabarreledness transfers from $A$ to $A_{e}$. The proof is straightforward.

Proposition 17. If $A$ is infrabarreled, so is $A_{e}$. 
In order to relate $P(A)$ to $P\left(A_{e}\right)$, we recall (Hewitt and Ross [7]) that an element $f$ of $P(A)$ is called extendable if it is hermitian and there exists $a \geqq 0$ such that

$$
|f(x)|^{2} \leqq a f\left(x^{*} x\right) \quad x \in A .
$$

If $f \in P(A)$ is extendable we define

$$
N(f)=\inf \left\{a \geqq 0:|f(x)|^{2} \leqq a f\left(x^{*} x\right) \forall x \in A\right\},
$$

and notice that $f=0$ if and only if $N(f)=0$. It is well-known that if $f \in P(A)$, then there exists $F \in P\left(A_{e}\right)$ which extends $f$ if and only if $f$ is extendable. In this case we must have $F(e) \geqq N(f)$.

Let us now assume that every element of $P(A)$ is extendable.

Proposition 18. If $P(A)$ is generating, so is $P\left(A_{e}\right)$.

Proof. If $F \in A_{e}^{\prime}$, then $\left.F\right|_{A} \in A^{\prime}$, so we can find $g$ in the linear span of $P(A)$ which equals $\left.F\right|_{A}$. Thus we can find $G$ in the linear span of $P\left(A_{e}\right)$ such that $\left.F\right|_{A}=g=\left.G\right|_{A}$. Thus $F-G \in A_{e}^{\prime}$ must be of the form $(F-G)(x+\lambda e)=\lambda \mu(x \in A, \lambda \in \mathbb{C})$ for some $\mu \in \mathbb{C}$. For any $\alpha \geqq 0$ the element $G_{\alpha}$ of $A_{e}^{\prime}$ defined by $G_{\alpha}(x+\lambda e)=\alpha \lambda$ belongs to $P\left(A_{e}\right)$. Thus $F-G$, and hence $F$, belongs to the linear span of $P\left(A_{e}\right)$.

If we introduce the following generalisations of properties $(C)$ and $\left(C^{\prime}\right)$ :

(GC) for any null sequence $\left(x_{n}\right)$ in $A$ we can find $\lambda>0$ such that $\lambda f\left(x_{n}^{*} x_{n}\right) \leqq N(f)$ for all $n \in \mathbb{N}$ and $f \in P(A)$;

(GC') for any $x \in A$ we can find $\lambda>0$ such that $\lambda f\left(x^{*} x\right) \leqq N(f)$ for all $f \in P(A)$, then simple calculations now show that:

Proposition 19. If $A$ satisfies (GC), then $A_{e}$ satisfies (C). If $A$ satisfies $\left(\mathrm{GC}^{\prime}\right)$, then $A_{e}$ satisfies $\left(\mathbf{C}^{\prime}\right)$.

Consequently, the results of Section 5 may be appealed to.

Theorem 20. If $(A, t)$ is a locally convex *-algebra such that every element of $P(A)$ is extendable, then $A$ is a pre-C*-algebra if and only if it satisfies properties (A),(B),(GC), and $A$ is a barreled pre-C $C^{*}$-algebra if and only if it satisfies properties $\left(\mathrm{A}^{\prime}\right),(\mathrm{B}),(\mathrm{GC})$.

Finally, let us consider under what circumstances every element of $P(A)$ is extendable. Let us suppose that the algebra $A$ possesses an approximate identity $\left(e_{a}\right)$. We say that $\left(e_{a}\right)$ is $C^{*}$-bounded if the net $\left(e_{a}^{*} e_{a}\right)$ is bounded. If $A$ is a pre- $C^{*}$-algebra, then bounded and $C^{*}$-bounded approximate identities are the same.

Proposition 21. If $A$ possesses a $C^{*}$-bounded approximate identity, or if $A$ is barreled and possesses a bounded approximate identity, then every element of $P(A)$ is extendable.

Proof. Let $\left(e_{\alpha}\right)$ be the approximate identity for $A$. Since $f\left(e_{a}^{*} x\right)=\overline{f\left(x^{*} e_{a}\right)}$ for all $f \in P(A), x \in A$ and all $\alpha$, it follows that every element of $P(A)$ is hermitian. For any 
$f \in P(A)$, the net $\left(f\left(e_{\alpha}^{*} e_{\alpha}\right)\right)$ is bounded. This is obvious if $\left(e_{\alpha}\right)$ is $C^{*}$-bounded. If $A$ is barreled and $\left(e_{\alpha}\right)$ is bounded, it follows from the fact that the map $x \mapsto f\left(x^{*} x\right)^{1 / 2}$ is a continuous seminorm on $A$ (Lassner [9]). Thus we can always find $a \geqq 0$ such that $f\left(e_{\alpha}^{*} e_{\alpha}\right) \leqq a$ for all $\alpha$, and so $\left|f\left(e_{\alpha}^{*} x\right)\right|^{2} \leqq f\left(e_{\alpha}^{*} e_{\alpha}\right) f\left(x^{*} x\right) \leqq a f\left(x^{*} x\right)$ for all $x \in A$ and all $\alpha$. Taking limits, we deduce that $f$ is extendable.

If $A$ is a $C^{*}$-algebra, then $A$ possesses a bounded approximate identity (Dixmier [5], 1.7.2). Thus we have the following results.

Theorem 22. If $A$ is a locally convex *-algebra such that:

(a) $A$ is infrabarreled;

(b) $P(A)$ is generating;

(c) A has a $C^{*}$-bounded approximate identity;

(d) for every null sequence $\left(x_{n}\right)$ in $A$ we can find $\lambda>0$ such that $\lambda f\left(x_{n}^{*} x_{n}\right) \leqq N(f)$ for all $f \in P(A)$ and $n \in \mathbb{N}$.

then $A$ is a pre- $C^{*}$-algebra.

Theorem 23. A locally convex *-algebra $A$ is a $C^{*}$-algebra if and only if:

(a) $A$ is sequentially complete;

(b) $A$ is barreled;

(c) $\mathrm{A}^{+}$is a normal cone in $A$;

(d) $A$ has a bounded approximate identity;

(e) for any $x \in A$ we can find $\lambda>0$ such that $\lambda f\left(x^{*} x\right) \leqq N(f)$ for all $f \in P(A)$.

The version of Theorem 23 for unital algebras is as follows:

Theorem 24. A unital locally convex *algebra $A$ is a $C^{*}$-algebra if and only if:

(a) $A$ is sequentially complete;

(b) $A$ is barreled;

(c) $A^{+}$is a normal cone in $A$;

(d) for any $x \in A$ we can find $\lambda>0$ such that $e-\lambda x^{*} x \in A^{+}$.

\section{REFERENCES}

1. J. Alcantara and D. A. Dubin, $I^{*}$-algebras and their applications, Publ. RIMS Kyoto Univ. 17 (1981), 179-199.

2. G. R. Allan, A spectral theory for locally convex algebras, Proc. London Math. Soc. (3) 15 (1965), 399-421.

3. G. R. Allan, A note on $B^{*}$-algebras, Math. Proc. Cambridge Philos. Soc. 61 (1965), 29-32.

4. F. F. Bonsall and J. Duncan, Numerical ranges of operators on normed spaces and of elements of normed algebras (L.M.S. Lecture Note Series 2, Cambridge University Press, 1971).

5. J. Dixmıer, $C^{*}$-algebras (North-Holland, Amsterdam, 1982). 
6. P. G. Dixon, Generalised $B^{*}$-algebras, Proc. London Math. Soc. (3) 21 (1970), 693-715.

7. E. HewitT and K. Ross, Abstract Harmonic Analysis (Springer-Verlag, Berlin, 1963).

8. Ky Fan, On infinite systems of linear inequalities, J. Math. Anal. Appl. 21 (1968), 475-478.

9. G. Lassner, Topological algebras of operators, Rep. Math. Phys. 3 (1972), 279-293.

10. S. SAKaI, $C^{*}$-algebras and $W^{*}$-algebras (Springer-Verlag, Berlin, 1971).

11. H. H. SCHAEFER, Topological Vector Spaces (Springer-Verlag, Berlin, 1971).

12. A. W. Woon, Numerical range and generalised $B^{*}$-algebras, Proc. London Math. Soc. (3) 34 (1977), 245-268.

Sidney Sussex College

CAmbridge

CB3 3HU 